\title{
A versatile capillaric microfluidics viscometer platform for bar-graph type point-of-care
} diagnostics

\author{
Claude Meffan ${ }^{1 *}$, Julian Menges ${ }^{1}$, Fabian Dolamore $^{2}$, Daniel Mak $^{3}$, Conan Fee ${ }^{2}$, \\ Volker Nock', Renwick C.J. Dobson ${ }^{4,5}$
}

Dr. Claude Meffan, E-mail: claude.meffan@canterbury.ac.nz

1 Biomolecular Interaction Centre, MacDiarmid Institute for Advanced Materials and Nanotechnology, and Department of Electrical and Computer Engineering, University of Canterbury, PO Box 4800, Christchurch 8140, New Zealand

2 Biomolecular Interaction Centre and School of Product Design, University of Canterbury, PO Box 4800, Christchurch 8140, New Zealand

3 Biomolecular Interaction Centre, Maurice Wilkins Centre for Biodiscovery, MacDiarmid Institute for Advanced Materials and Nanotechnology, and School of Biological Sciences, University of Canterbury, PO Box 4800, Christchurch 8140, New Zealand

4 Bio21 Molecular Science and Biotechnology Institute, Department of Biochemistry and Molecular Biology, University of Melbourne, Parkville, Victoria 3010, Australia.

Keywords: Capillaric Circuits, Microfluidics, Rheology, capillaric-Field Effect Transistor, Lab-on-a-Chip

Abstract

A novel capillary action microfluidic viscometer has been designed that can measure the relative viscosity of a sample compared to a control liquid. Using capillary action circuits, the viscosity of a sample is transformed into a microfluidic bar-graph format without the use of external instrumentation. The bars in this case are represented by the distance that a liquid has flown through a microfluidic channel, relative to another liquid in an identical channel. As the device does not require external instrumentation, its use is targeted at point-of-care (PoC) situations. This implementation is made practical through capillaric Field Effect Transistors, and the conditional flow paths they enable. In this paper, we report on the design, operation, and performance of a two-channel version viscometer device exclusively based on capillary 
action circuits. Using poly-ethylene glycol solutions as viscous samples, we demonstrate that the device can transduce the relative viscosity consistently to within $2 \%$. Enabled by the flexibility of the capillary action circuits, we additionally present a modified device which can measure transparent liquids without the need to add colorants to the sample. The forms of the device presented in this work have applications in both medical care and scientific measurements - particularly for PoC measurements. 


\section{Introduction}

Point-of-care diagnostic devices are a key application of microfluidics. In general, point-of-care (PoC) devices reduce the instrumentation complexity of measurements, enabling the transfer of clinical laboratory procedures into simplified form factors. In its ideal form, this allows untrained personnel to conduct measurements outside the laboratory environment. As previously reported, $\mathrm{PoC}$ devices promise to assist with hospital triage, ${ }^{[1]}$ help with remote diagnosis, ${ }^{[2,3]}$ improve certain clinical outcomes, ${ }^{[2]}$ reduce the length of hospital stays, ${ }^{[4]}$ and further equity and satisfaction with medical care for rural communities. ${ }^{[3,5,6]}$ As such, there is major societal, ethical, and commercial interest in extending the already available PoC toolkit towards a wider range of functionalities.

While PoC devices in general do improve the turnaround times of medical results, improved clinical outcomes are not universally demonstrated. ${ }^{[2]}$ This fact hints at the uncompromising cost, ${ }^{[4]}$ complexity of use, and consistency ${ }^{[7]}$ requirements which $\mathrm{PoC}$ devices must adhere to in order to positively influence medical outcomes.

Self-driven capillary circuits are one promising approach for $\mathrm{PoC}$, as they do not require electrical accessories and can be pre-programmed through their geometry. ${ }^{[8]}$ Recently we introduced a novel valve and its use for the design of complex capillaric circuit components including integrated logic and valving. ${ }^{[9]}$ Here, we demonstrate the combination of these components to yield a simple-to-read assay, specifically designed for use in PoC applications.

As a target parameter, the current work focuses on the measurement of rheological properties of biological fluids. A notable example of this is the viscosity of blood, ${ }^{[10,11]}$ which can be used as a therapeutic management method for people with hematological and circularity disorders, but also in the diagnosis and management of disease. Trauma, inflammation, and malignancy are all known to create alterations to a range of protein concentrations in blood plasma and therefore a shift in the viscosity. ${ }^{[12]}$ Up to now, very few viscosity diagnostic tools have proven their potential for PoC diagnostics despite the appeal of the principle. Microfluidic viscometry on the other hand has been extensively explored in the past ${ }^{[13,14]}$, with established approaches based on capillary flow, ${ }^{[14-17]}$ tight junctions, ${ }^{[18,19]}$ and stagnation flow. ${ }^{[20,21]}$ These microfluidic rheology devices commonly require external pumps and pressure chambers, ${ }^{[19,21-23]}$ external measurement equipment, ${ }^{[23,24]}$ internal sensors, ${ }^{[15,16]}$ or a combination of these elements. 
Although this results in a high level of precision, applicability for a wide range of liquids and fast measurement time, the resulting platforms are still fundamentally advanced laboratory equipment ${ }^{[25]}$ and generally do not meet all that is required of a true $\mathrm{PoC}$ device. In addition to the requirements discussed above, viscosity measurement tools must also overcome the temperature dependency of viscosity. In a PoC test, the environmental temperature may be highly variable, and thus can create a substantial variation in the result.

Based on this discussion, the challenges in creating a medical PoC viscometer can be defined as sufficiently high accuracy, high consistency, cost-efficiency, temperature independence, minimal or no external instrumentation, ease of use (easy to perform steps) and readability of the result (minimum information, high visibility).

We suggest that a co-flow stream viscometer ${ }^{[26,27]}$ design implemented using capillaric circuits $^{[8,28]}$ represents the best approach to address these challenges. Such co-stream viscometers represent the relative properties of a sample against a reference. In addition, they have the potential to self-adjust for temperature effects. ${ }^{[29]}$ The use of capillaric circuits means the viscometers also do not require complex pumping systems, due to the capillary forces used, and simple data representation through flow distance measurement can be implemented. ${ }^{[17]}$ The latter can be read-out without the need for external measurement equipment, although optical measurement is not necessarily excluded.

One example of a microfluidic device that has demonstrated many of the requirements above was presented recently. ${ }^{[27]}$ In this device, a sample and reference liquid enter a single channel simultaneously. After the liquids have entered, the channel divides into a fan like structure. Flowing at a rate proportional to their viscosity, the liquids create a speedometer-like readout for the relative material properties. This data representation is intuitive, and completely fulfills the requirements for ease of interoperability. Potential shortcomings are that this approach is strongly linked to production quality and orientation, as well as the need of a stationary flow regime and thus large volumes. Deviation in the height of the fanned section, due to roof sagging, or general manufacturing variation could cause substantial errors in read-out. 
Here, we present a novel approach for a capillary co-stream viscometer that is built to be integrated into any kind of capillary-action driven PoC device. The design targets a bar graph type format where the distance a sample flows in a channel represents the viscosity. ${ }^{[17,30-32]}$ This allows relative properties of the two materials to be presented by the relative lengths of parallel bars. Conceptually, this is demonstrated in Figure 1. The bar graph format is common, well understood and known to clearly convey numerical results (as compared to trend results). ${ }^{[33]}$ In

a) Liquid Input

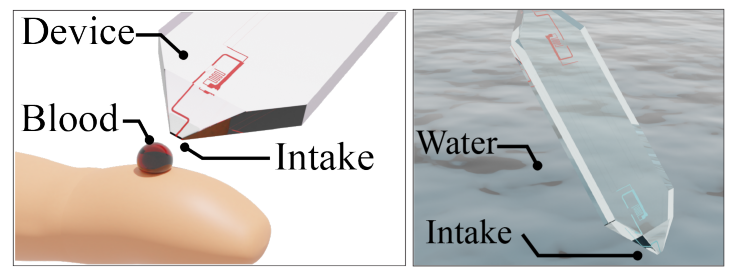

b) Automated (hidden) operations

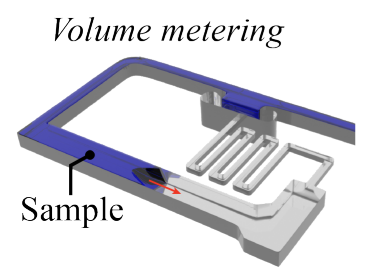

Valving and isolation
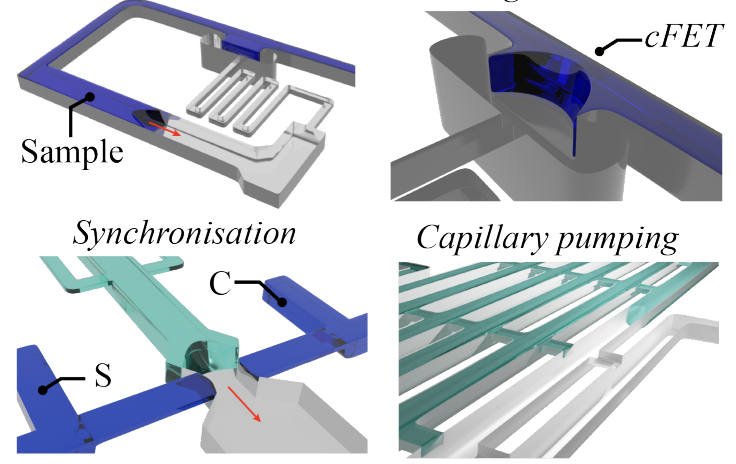

c) Result presentation

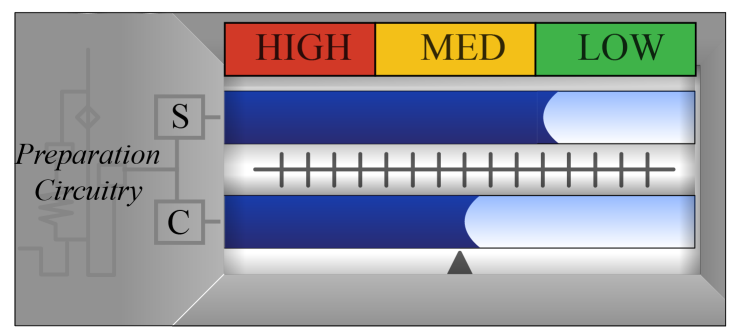

Figure 1: Concept of the bar-graph viscometer. The device uses capillary pumps to transduce the relative viscosities of a sample (S) and control (C) liquid into two bar lengths. (a) The user directly inputs a sample to be measured, for example, blood. The user then fills the device with water which commences the measurement. These are the only steps required by the user. (b) Following the user input stage, all necessary steps to carry out the viscosity measurement are automated by the capillaric circuit. The automated steps include precise sample volume metering, fluidic valving, and channel isolation. After the sample $(S)$ and control $(C)$ materials are prepared, the pumping is commenced for each material at the same time. (c) After the capillary pump is expended, the result of the measurement can be interpreted from the different lengths of two bars. In this example liquid $\boldsymbol{S}$ has a viscosity $30 \%$ less than $\boldsymbol{C}$. 
our case the bar-graph format viscometer is enabled through the use of capillaric Field Effect Transistors (cFETs), ${ }^{[9,34,35]}$ which allow for conditional flow paths in the circuit layout to be practically implemented.

In the following we demonstrate the core function of the bar-graph type flow stream viscometer and show that effects of temperature are mitigated by the design. Additionally, we show an extension of the bar-graph co-stream viscometer that separates the read-out and sensing functions, which is useful for the measurement of uncolored samples. Both designs make use of the recently introduced cFETs, ${ }^{[9]}$ which allow for fully integrated sequential control of all necessary circuit operations without the need for external devices. This includes operations such as metered reservoir charging and sealing, prefilling of the measurement circuitry and timed initiation, all which can be preprogrammed into the device design. The particular design of the viscometer includes resistors $(-M-),{ }^{[36]}$ capillary pumps $(\square \square),{ }^{[37]}$ reservoirs $(-\square-),{ }^{[28]}$ two

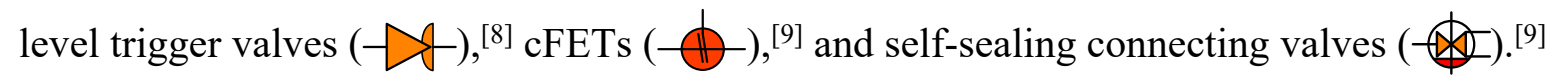

\section{Bar-graph co-stream viscometer}

\subsection{Circuit Design}

When an equal driving pressure is applied to two liquid samples in identical micro-channels the flow rate in each channel will be proportional to the viscosity of each liquid. ${ }^{[36]}$ Due to this, the distance over which a liquid flows in a given time will reflect the rheological properties of the liquid. This simple principle is the core mechanism behind the bar-chart format co-stream viscometer.

To implement this format, a uniform pressure needs to be applied to both sample and control simultaneously. Existing microfluidic rheometer systems use external pumps or manually actuated pressure chambers to apply a known and consistent pressure, often in combination with external measurement systems. ${ }^{[19,21-23]}$. In this work, we instead use the Laplace pressure created by a single liquids' meniscus to apply the driving pressure to both the sample and control under test. ${ }^{[8,28,37]}$ This allows the co-stream viscometer to operate without the need for external pumps. The Laplace pressure exerted by a meniscus can be calculated using the YoungLaplace Equation (1): 


$$
P_{\text {cap }}=-\gamma\left(\frac{1}{R_{\text {horizontal }}}+\frac{1}{R_{\text {vertical }}}\right)
$$

where $P_{\text {cap }}$ is the capillary pressure, and $R_{\text {horizontal }}$ and $R_{\text {vertical }}$ are the primary radii of the meniscus in the horizontal and vertical direction, respectively.

Many existing capillary viscometer devices use the self-pumping of a single liquid over time to measure the rheology, ${ }^{[24]}$ however a co-stream or relative viscometer requires two liquids. The simplest co-stream capillary viscometer that could be envisaged would use each sample liquid to perform self-pumping in separate, but identical, capillary channels. While we tested devices with such channels, these did not perform well for the purpose of viscosity measurements as variations in surface wetting create additional degrees of freedom and therefore error in the system. Purely capillaric viscometer systems up until now have generally required the contact angle/capillary pressure to be measured and fitted beforehand or in-situ. ${ }^{[14,15]}$

To circumvent this problem, a circuit was designed in which the samples themselves did not perform self-pumping. Instead, one capillary pump, operating on a single "working fluid", draws the two sample liquids through identical flow resistances. This creates a single capillary pressure that is applied equally to both liquid samples and, as a result, allows a representative measurement to be made without the need to know each liquid's wetting properties. The flow rate that results is a function of channel geometry, temperature, and the liquids viscosity. By design, the channel geometry is identical, so when the device and tested liquids are at the same temperature, the result will reliably represent the relative viscosity of the liquids. A capillaric circuit diagram of the design is shown in Figure 2(a). The system is composed of a self-metering sample intake circuit, a transducing flow resistance, a capillary pump, and a liquid switch that connects the latter with the transducing circuitry. The circuit diagram of the sample in-take subcircuit is shown in Figure 2(b). For notational convenience and to provide design flexibility, the combination of the sample intake sub-circuit, bypass line, and transducing resistor can be combined into a functional block labelled B1 in Figure 2(b). The functional block B1 is connected to other parallel or series functional blocks, as well as a self-sealing connection valve $^{[9]}$ and capillary pump, to implement the desired viscometer function. 
a)

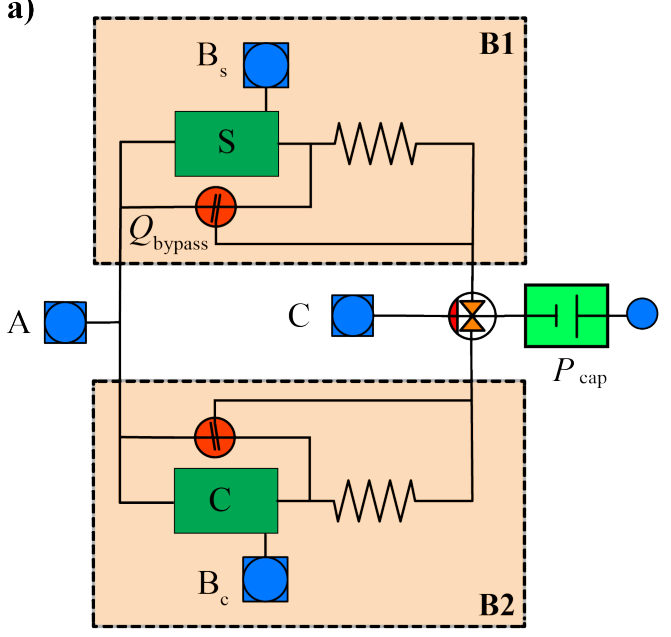

b)

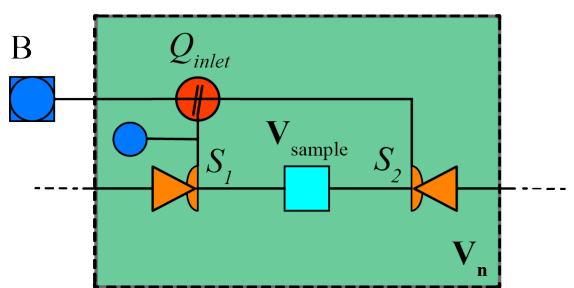

c)

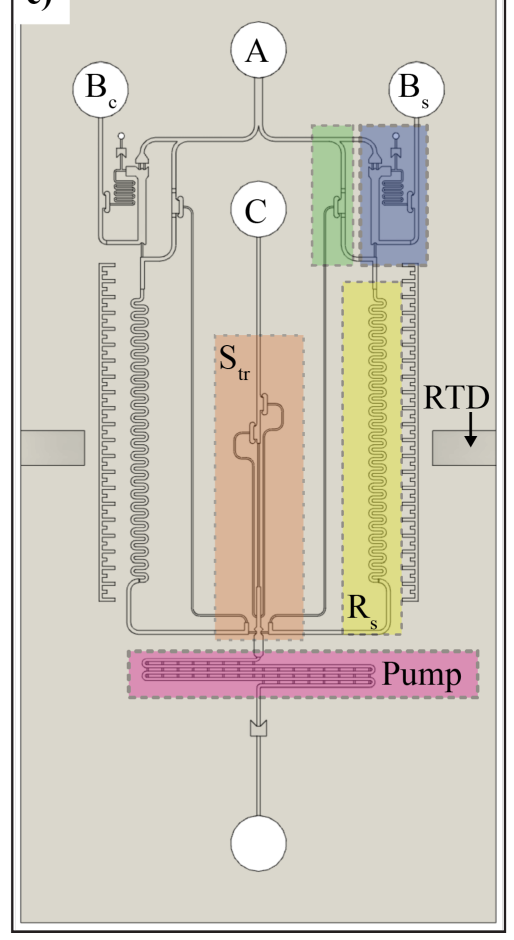

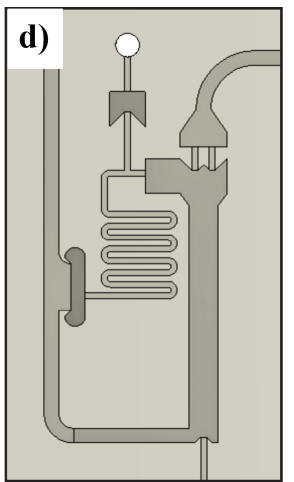

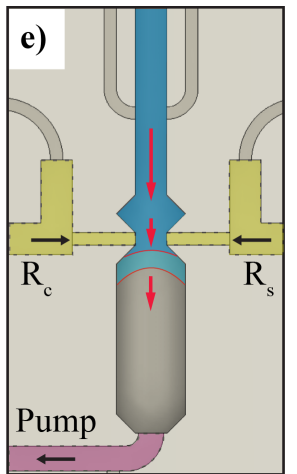

Figure 2: Capillaric circuit and computer aided design diagrams of the co-stream capillary viscometer. (a) The proposed device compares the rheological properties of a sample $(\boldsymbol{S})$ and a well characterized control liquid $(\boldsymbol{C})$. Note that in (a) the sample intake circuitry is abstracted for simplicity. It is shown as a full capillaric circuit in (b). In this circuit $\boldsymbol{A}$ is the water supply inlet, $\boldsymbol{B}_{\boldsymbol{s}}$ the sample inlet, $\boldsymbol{B}_{\boldsymbol{c}}$ the control inlet, $\boldsymbol{C}$ the trigger inlet, $\boldsymbol{P}_{\text {cap }}$ is a capillary pump, $\boldsymbol{S}$ and $\boldsymbol{C}$ are the sample and control reservoirs respectively, and $\boldsymbol{Q}_{\text {bypass }}$ is a cFET which seals the sample chamber bypass line. (b) Capillaric circuit diagram showing the function of the sample intake block. The circuit intakes a sample from inlet $\boldsymbol{B}$ and fills a reservoir $\boldsymbol{V}_{\text {sample. This reservoir is situated }}$ between two trigger valves $\boldsymbol{S}_{1}$ and $\boldsymbol{S}_{2}$, which connect the reservoir in series with remaining circuitry. After the reservoir is full, the inlet $\boldsymbol{B}$ is sealed from the reservoir using the $\boldsymbol{Q}_{\text {inlet }} c F E T$. (c) Physical device structure with labels indicating the capillary pump (pink), transducing resistance $R_{s}$ (yellow), self-sealing connection valve $\boldsymbol{S}_{\text {tr }}$ (orange), the sample bypass line (green), and the sample intake circuitry (blue). The recess for the thermometers is marked as RTD. As the device is symmetrical, only the control side of the device is unannotated for visual clarity. (d) Detailed view of the self-sealing sample intake circuitry used for both the sample and control. (e) Detailed view of the self-sealing connecting valve, $S_{t r}$ where it interfaces with transducing resistances. The point where the transducing resistors merge with the connecting valve is referred to as the release junction. Pink and yellow indicate connections to other parts of the circuit. The blue area illustrates how a liquid front traverses through the junction, while the red arrow indicates direction and magnitude of fluid flow due to the modulation of capillary pressure by the changing wall angle.

For a basic co-stream viscometer, two liquids are required. As such two parallel functional blocks, $\mathbf{B} 1$ and $\mathbf{B} 2$ are used. This configuration measures the viscosity of a single sample in B1 against a control sample in B2. If more than two liquids, or a combination of liquids were desired, the system can be extended simply by adding further identical functional blocks in parallel or series combinations with the existing system. In addition to the basic two block system, we also demonstrate a series block combination in this work. This design allows for the use of a high-contrast read-out fluid for visualization, instead of the relying on the analyte coloration itself for readout. 


\subsection{Device operation and testing}

An annotated computer aided design (CAD) rendering is presented in Figure 2(c). The key elements are the sample intake circuitry (blue), sample bypass line (green), transducing flow resistance (yellow), self-sealing connecting valve (orange) and capillary pump (pink). Also annotated are the water supply inlet $A$, sample inlet $B_{s}$, control liquid inlet $B_{c}$, and trigger inlet $C$. Figure 2(d) shows a detailed view of the sample intake circuitry, which intakes a defined volume of sample independent of user action, and places it in line with a water supply and transducing resistance. A detailed view of the subsequent release junction is shown in Figure 2(e), with the shaded yellow and pink regions indicating connections to the flow resistances and pump. Blue color schematically illustrates how water self-wets through this junction. The geometry of the release junction itself widens immediately after the triggering liquid front connects to the transducing resistance regions. This reduces the Laplace pressure of the liquid front, slowing its progress, and allowing the self-sealing connecting valve time to close. As a result, the consistency of the volume displaced through the transducing resistors is increased substantially when compared to designs without this geometry.

For the purpose of demonstrating the operation of the prototype device the user input is currently divided into three stages of hand-pipetting. However, simple design changes would enable a future version of the device to directly receive the analyte from a user (in a similar manner to existing blood glucose testing strips) or a dispensing robot for integration in a largescale laboratory test setting. The user input can be summarized into the three stages of water supply intake, followed by sample/control liquid intake, and finally pump switching/activation. In a production device, the final stage could be further automated through the capillaric circuit structure. This could be achieved by connecting the triggering input, $\mathrm{C}$, to the water supply through a large flow resistor to time the triggering event. Similarly, the control sample would not need to be manually input, as it may instead be created on the device by mixing water from the supply with solid reagents. ${ }^{[9]}$ Figure 3(a), (b) and (c) show an experimental demonstration of the stages of operational input described above. A video summarizing the general operation of the device is provided in Video S1 (Supplementary Information). An example of the type of final bar graph-type result corresponding to two samples of differing viscosities is shown in Figure 3(d). The bar-length in this case is represented by the distance the dark blue colour has flown through the serpentine flow resistors on the sample and control sides. 

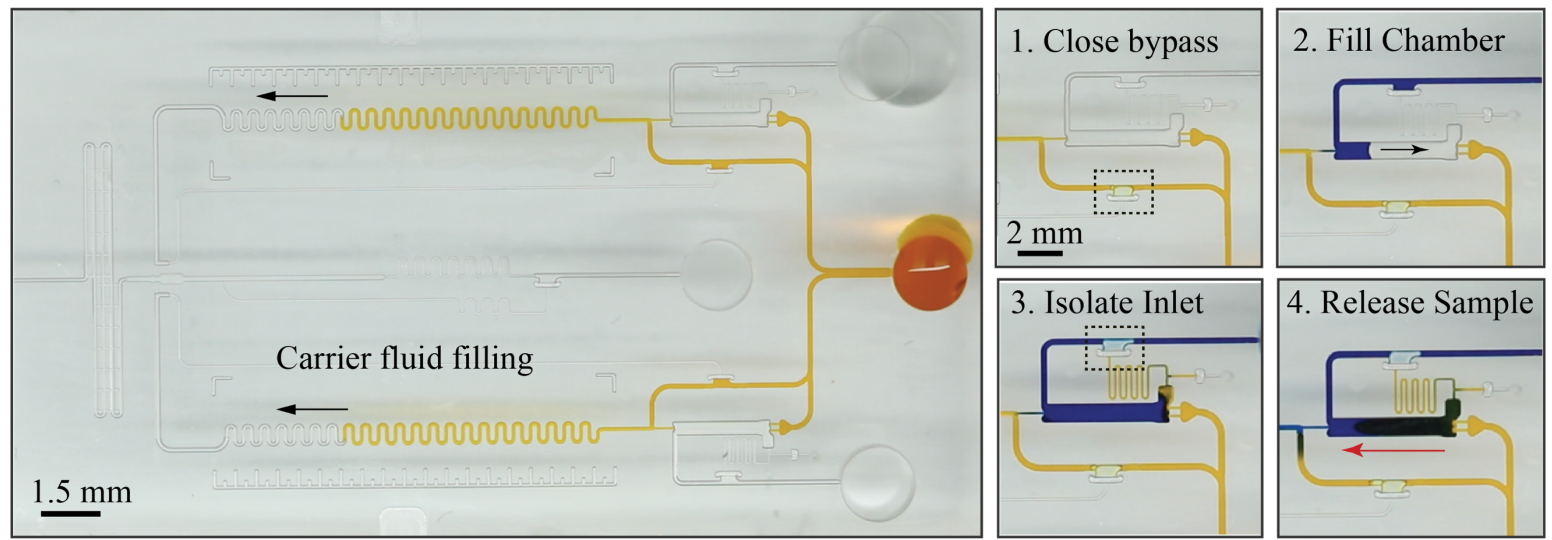

\section{c) Triggering}

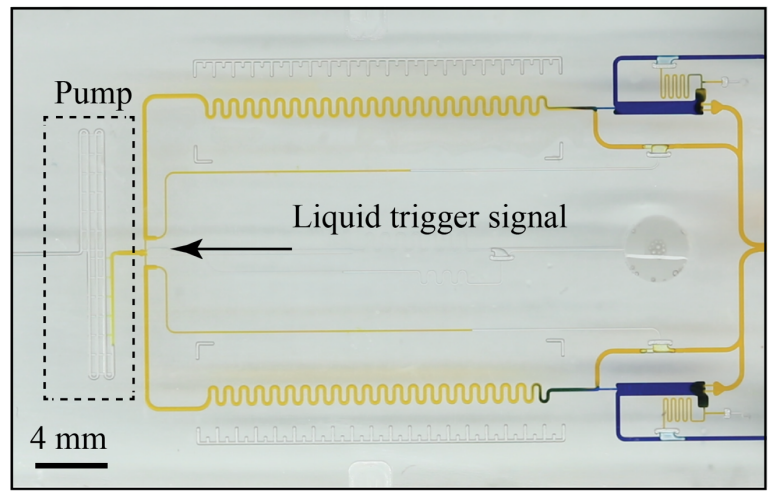

d) Measurement

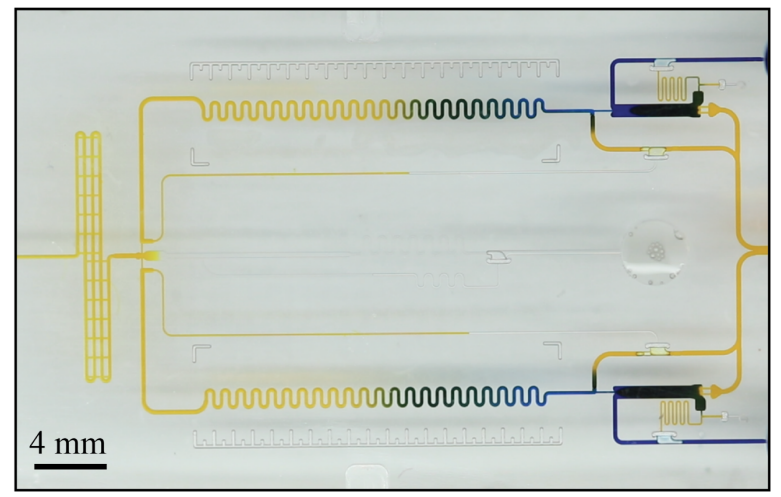

Figure 3: Basic operation of the co-stream differential viscometer. (a) Working liquid (dyed water in this case) bypasses the sample chamber and fills the transducing flow resistors up until the release junction of the self-sealing connecting valve. (b) Following this, the self-metering sample/control chamber is filled. In 1, the bypass line is sealed using a cFET. In 2, the sample chamber is filled with the viscous sample. In 3, the inlet cFET is actuated, sealing off the sample inlet from the volume-controlled chamber. Finally, 4 shows the sample chamber after an experiment has been carried out. (c) An experiment is commenced by applying liquid to the triggering inlet. This liquid front from the triggering inlet connects the flow resistors to the capillary pump. Two cFETs seal the triggering inlet from the pump, meaning fluid is only displaced through the fluid resistors. (d) Once the capillary pump is expended, the result can be read from the lengths shown by the flow resistors. When compared to the control resistor, the distance the sample flows in the loop will be an inverse function of its viscosity.

The steps to operate the device can be described using the circuit elements defined by Figure 2 (a)-(e) and operational stages outlined in Figure 3. In step one, water (acting as the working fluid) is added to the water-supply inlet " $\mathbf{A}$ ". Under capillary self-pumping the working fluid flows through the sample bypass lines and fills the transducing flow resistances up until the release junction, $S_{t r}$. While this happens, the two-level trigger valves $S_{1}$ and $S_{2}$ prevent the working fluid from flowing into the sample chamber. Once the transducing resistance $R_{s}$ is filled, a feedback line from the release junction actuates the $Q_{\text {bypass }} \mathrm{cFET}$. This permanently closes the sample bypass line. By actuating the bypass cFET from the release junction, the sample bypass line only closes when the sample flow resistances are completely full, completing step one of the operation. 
In step two, the sample and control liquids are loaded into the sample reservoirs. The goal of the sample inlet circuitry is to place a known volume of liquid in series with the transducing resistances while not inducing unwanted flow or requiring precise volume input by the user. ${ }^{[35]}$ While spurious fluid flow in this step could dilute or corrupt samples and change their rheology, a requirement of precise user input would limit uptake for PoC applications. The step-by-step operation of the sample intake circuitry is shown in Figure 3(b), with Figure 3(b)-1 representing the initial state. It should be noted that $Q_{\text {bypass }}$ is already closed at this stage from step one. In Figure 3(b)-2, liquid is placed into the $B_{s}$ sample inlet and drawn into the chamber through capillary self-pumping. Due to the high meniscus pressure of both the $Q_{\text {bypass }}$ occluding bubble ${ }^{[9]}$ and the release junction trigger valve $\left(S_{t r}\right)$, very little spurious flow is drawn from the already filled sections of circuitry. Instead, the chamber is filled entirely from the $B_{s}$ inlet. In Figure 3(b)-3, the sample chamber is now full, and the $Q_{\text {inlet }}$ cFET is actuated. This isolates the $B_{s}$ inlet from the chamber. As a result of this process, a volume-controlled sample chamber is now in series with the transducing flow resistance and the water supply through the two-level trigger valves $S_{1}$ and $S_{2}$. This procedure is repeated for the control sample. Figure 3(b)-4 shows and example of the sample chamber after the volume has been dispensed into the flow resistor.

In step three, shown in Figure 3(c), water is applied to the triggering inlet, $C$. When liquid reaches the release junction, it triggers the two-level trigger valve $S_{t r}$ on both sides of the device simultaneously. This connects the sample and control transducing resistances to the capillary pump and commences the measurement. While this happens, the " $\mathbf{C}$ " release inlet itself is isolated from the capillary pump using two series cFETs $Q_{t r l}$ and $Q_{t r 2}$. The capillary pump now draws the sample and control liquids from the sample reservoirs through their respective flow resistors.

In this example, the capillary pump volume was designed so that two equal viscosity liquids would be pulled halfway through each transducing resistor. An example of a differing viscosity measurement is shown in Figure 3(d), where the result is read out by simply recording the length ratio of the two blue bars. A shortening of one bar, with respect to the other, represents a corresponding increase in viscosity. For a PoC measurement, this read out would be aided by a graphic overlay which would allow the result to be interpreted without computation. One option for a visual overlay would be a linear classification type scale which shows "HIGH-MED-LOW" ranges (see Figure 1(c)). Alternatively, an additional ruler overlay would allow a physician or trained user to obtain a numerical value. 
For demonstration purposes, the colored bar lengths in the current work were measured using image processing tools (ImageJ) ${ }^{[38]}$ to obtain a precise result. However, the image processing utilized for this is very simple and could easily be carried out using a smartphone application if required.

\subsection{Viscous sample testing}

To test the function of the bar-graph type viscometer device, a range of solutions with wellcontrolled fluid viscosities were prepared. The selected samples were composed of five volumetric mixes of stock concentration $2 \mathrm{kDa}$ and $4 \mathrm{kDa}$ molecular weight poly-(ethyleneglycol) (PEG) (stock concentration 50\% with water, Sigma Aldrich). The concentrations tested were $\left(2 \mathrm{kDa}_{(\mathrm{x})}: 4 \mathrm{kDa}_{(1-\mathrm{x})}\right)$ for $\mathrm{x} \in\{1.0,0.75,0.5,0.25,0.0\}$. These volumetric mixtures created an almost linear viscosity distribution, spanning between that of $2 \mathrm{kDa}$ and $4 \mathrm{kDa}$ PEG. For this work, the control liquid was stock $2 \mathrm{kDa}$ PEG solution (stock 50\% concentration in Water, Sigma Aldrich). For reference values, the PEG mixes were measured with a conventional coneplate rheometer (MCR 302, Anton-Paar) over a temperature range of $15-45^{\circ} \mathrm{C}$. The full results of the cone-plate viscometer are included in Figure S1 (Supporting Information).

To test the design, the device was made hydrophilic by exposure to oxygen plasma and sealed against a hydrophobic polydimethylsiloxane (PDMS) cover using a spring-loaded device clamp (full process described in the experimental section). Following this, liquids were pipetted into the inlets and the movement of liquids in the devices recorded from above. The device sealing clamp and overhead filming set-up are shown in Figure S2 (Supporting Information).

Photographs of an example measurement performed with the bar-graph type co-stream viscometer are shown in Figure S3 (Supporting Information) and the results obtained from all measurements are summarized in Figure 4(a). A video visualizing the viscosity sample experiments is provided in Video S2 (Supplementary Information). The y-axis of Figure 4(a) shows the extent to which the viscous samples filled their respective transducing resistances as a percentage. Each viscous sample was tested three times. The average of the tests is presented as solid markers, and standard deviation is shown by the shaded areas. In addition, a linear regression was fitted and is shown as the dashed line. As anticipated, there is an equal and opposite linear trend between the sample and control results resulting from one fluid flowing fast than the other. From Figure 4(a) it can be further observed that there is a significant 

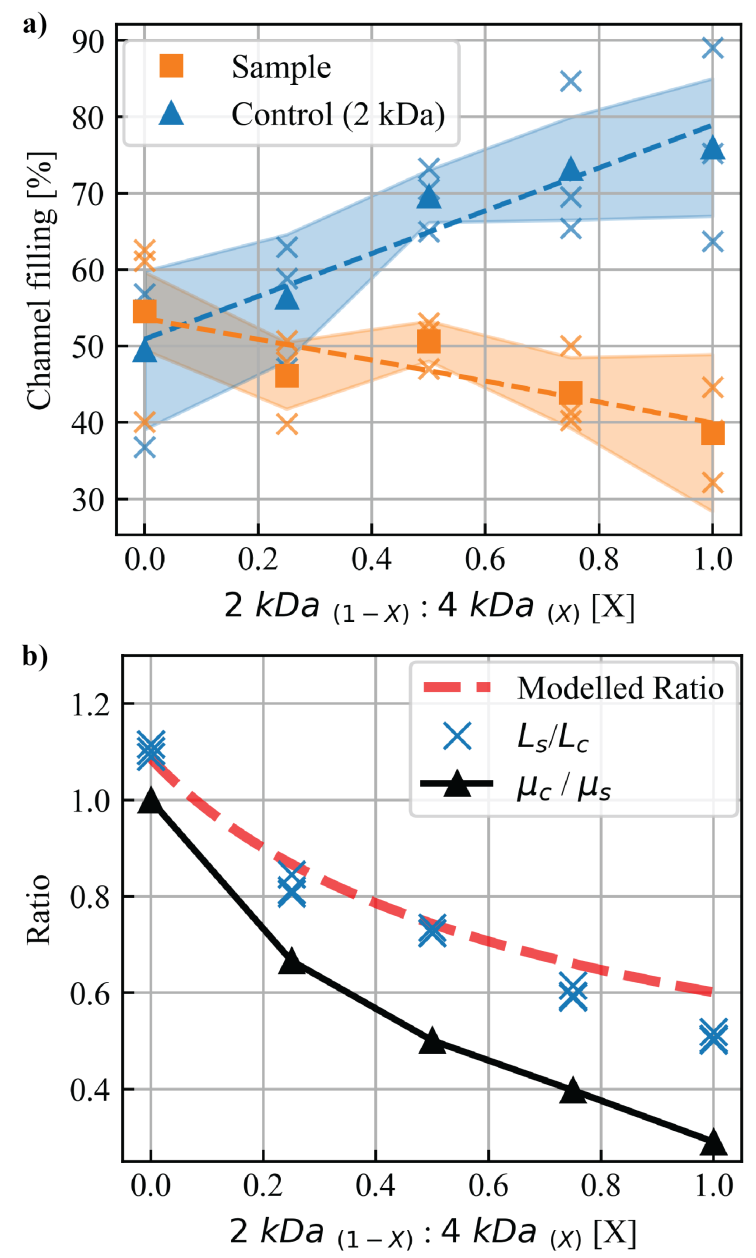

Figure 4: Experimental characterization of the differential viscometer using volumetric mixes of $2 \mathrm{kDa}$ and $4 \mathrm{kDa}$ Poly(Ethylene Glycol). (a) Plot of the percent each channel filled as a function of the total channel displacement. As the solutions became more viscous, the total displacement reduced. (b) Plot of the length ratio of the two bars representing the co-stream viscometer measurement. Also shown are the modelled fluid flow for the device and cone-plate rheometer results. Because fluid flow is inversely proportional to viscosity, the sample/control ratio is inverted when comparing bar-length to the measured viscosity of the materials.

variation in the total filling percentage between comparable experiments. This is caused by the variation in the sealing time of the connecting valve, $\mathrm{S}_{\mathrm{tr}}$, and contact angle variation. However, the intended function of the viscometer is to report the ratio of these results, not the absolute value. Such a ratio of the sample bar length, $\mathrm{L}_{\mathrm{s}}$, to the control bar length, $\mathrm{L}_{\mathrm{c}}$, is shown in Figure 4(b). As can be observed, when the ratio is recorded, the deviation between runs is significantly reduced. This maximum standard deviation in the result was less than $2 \%$ when reported as a ratio. As well as demonstrating the excellent precision of the device, this also illustrates the ability of co-stream viscometer to reduce major disturbances that might otherwise affect a viscometry measurement. 
Figure 4(b) also shows the reference ratio of the sample and control viscosities $\left(\mu_{\mathrm{c}} / \mu_{\mathrm{s}}\right)$, as measured by the cone-plate rheometer $(\boldsymbol{\Delta})$. Because flow in the capillaric circuits resistors is inversely proportional to viscosity, the ratio of sample-to-control is used to compare to the coneplate rheometer $\mu_{\mathrm{c}} / \mu_{\mathrm{s}}$ result. While there is a considerable offset between the capillary circuit bar-lengths and true viscosity ratio shown in Figure 4(b), this offset can be explained as a combination of the fabrication procedure and the contribution of the working fluid hydraulic resistance. On closer examination, the offset comprises two components, a constant offset in flow resistance, and an offset which increases with the difference between the sample and control viscosity. The constant offset is observed because of the length difference reported even when the sample and control liquids are identical. It is likely caused by geometrical errors induced as a side effect of the milling process used for device prototyping. ${ }^{[9]}$ The exact geometric error is not fully understood at this stage, however axis run-out, ${ }^{[39]}$ machine vibration, tool deflection may be potential sources. ${ }^{[40]}$ This offset could readily be removed in a production device through calibration. In addition to the offset caused by geometric error, there is an offset which increases with the difference between the sample and control viscosity. This offset can be attributed to the working fluid's contribution to the total system resistance. By example, when an experiment commences the hydraulic resistance of the transducing resistor is initially entirely defined by the working fluid. As the experiment proceeds, water is displaced and sample in-flows. This causes the sample resistance contribution to increase, and the working fluid contribution to decrease towards zero. This type of two-fluid model is commonly used in capillary microfluidic systems. ${ }^{[41]}$ In a situation where the fluids flow at identical rates, there is little difference between the ideal and reported result. However, when the sample and control flow at different rates, then the working fluid contribution disappears at different rates, causing deviation from the desired viscosity ratio result. Using these two offset sources, the evolution of the length ratio over time, $r(t)$ can be described using Equation 2.

$$
r(t)=\frac{x(t)}{y(t)}=D \times\left(\frac{\mu_{c}-\mu_{w}}{\mu_{s}-\mu_{w}}\right) \times\left(\frac{-\mu_{w} l+\sqrt{\left(\mu_{w} l\right)^{2}+2\left(\mu_{s}-\mu_{w}\right) t}}{-\mu_{w} l+\sqrt{\left(\mu_{w} l\right)^{2}+2\left(\mu_{c}-\mu_{w}\right) t}}\right)
$$

Where $x$ and $y$ are the distance a sample flows through the sample and control resistors respectively, $D$ is a constant factor representing inhomogeneities between the two fabricated resistances, $t$ is non-dimensional time, $l$ is the length of the transducing resistances, and $\mu_{\mathrm{c}}, \mu_{\mathrm{s}}$, and $\mu_{\mathrm{w}}$ are the viscosities of the control, sample, and working fluid, respectively. A derivation and in-depth analysis of Equation 2 are given in Figure S3 and Equations S1 (Supporting Information). Figure 4(b) shows the modelled fluid flow alongside the experimental data 
obtained using the bar-graph viscometer (red dotted line). For this, Equation 2 was evaluated with $\mathrm{D}=1.11,1=50 \mathrm{~mm}, \mu_{w}=1 \mathrm{mPa} . \mathrm{s}, \mu_{c}=35 \mathrm{mPa} . \mathrm{s}, \mathrm{t}=5 \times 10^{-5}$, and $35<\mu_{s}<115 \mathrm{mPa} . \mathrm{s}$ to reflect the cone plate rheometer measurements. The evaluated value of non-dimensional time was chosen such that the average sample or control displacement approximately matched the experimental results $(x, y \approx 30 \mathrm{~mm})$. As can be observed in Figure 4(b), this model closely aligns with the experimental results, validating the assumptions made regarding the source of the offset. In a future device, this offset may be reduced by adding an increased source resistance to the sample chamber. This would allow the resistance contribution of the sample or control to dominate the total resistance very quickly, and thus minimize the integrated error caused by the working fluid.

Even without such structural adjustments, the reported result can be calibrated or controlled for using Equation 2, albeit requiring an assumption about or measurement of the viscosity of the working fluid. If the viscous samples and working fluid are sufficiently separated in the absolute value of their viscosity, this may be an acceptable approach to calibrate the device. It should be noted that, in absence of a calibration, the error source is also minimized if the difference between the sample and the working fluid viscosities would be increased. Similarly, shortened transducing resistances would also reduce the error, but doing so would significantly increase the required tolerance on the sealing time of the self-sealing connecting valve, $S_{t r}$.

\subsection{Disturbance insensitivity}

One strength of co-stream viscometers is their use of differential rheology readout. ${ }^{[27]}$ In some cases this differential read-out reduces the impact of common disturbance forces, such as temperature. In the case where a disturbance force creates a proportional and equal change to the sample and control's viscosity, the differential result will be unchanged. For viscometers, and generally for rheological analytes, the most influential disturbance is the environmental temperature, since viscosity is highly dependent on this influence. ${ }^{[13]}$ 
a)

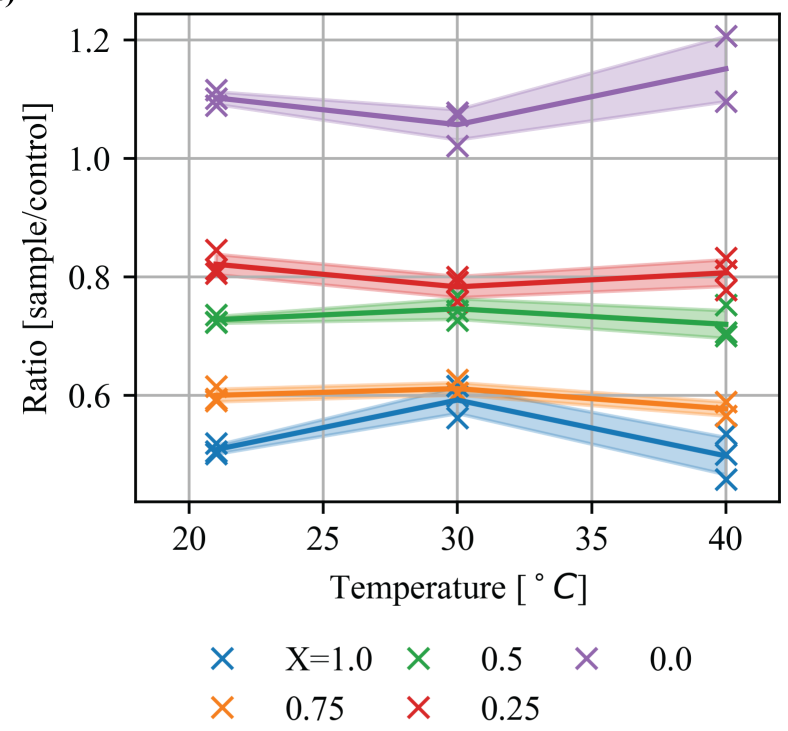

b)

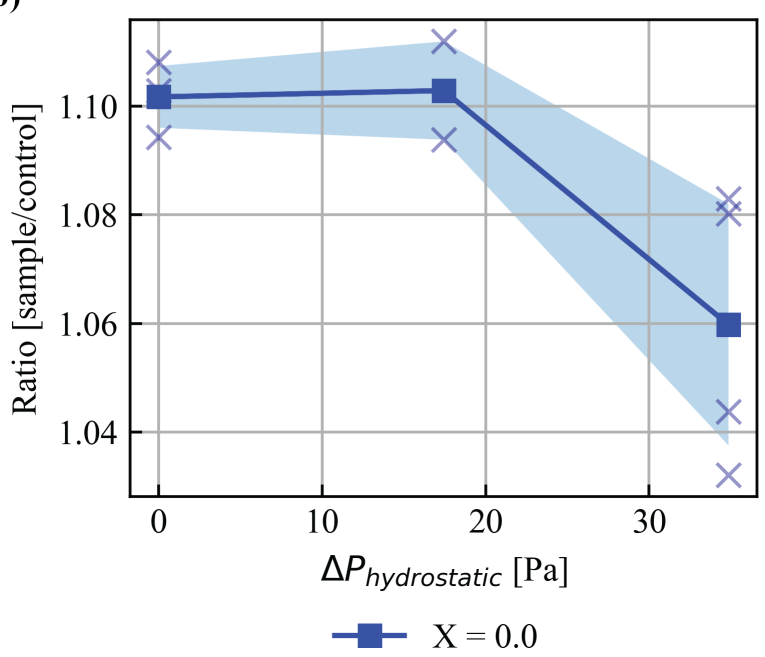

Figure 5: Common mode disturbance rejection capability of the bar-graph type co-stream viscometer. (a) Plot showing the temperature dependence of the results obtained with the device. Despite the absolute viscometry more than halving over this temperature range, the output consistently represents the relative properties. (b) Plot of the change in reported output as a function of differential hydrostatic pressure on the sample inlets.

To assess the temperature rejection of the bar-graph viscometer developed in this work, the device was mounted in a custom device clamp with an integrated Peltier stage in the base. A photograph of the device clamp is shown in Figure S2 (Supporting Information). With the device assembly mounted in the clamp, power was applied to the Peltier modules and the temperature of the device measured. The temperature measurement was made using a PT-100 platinum resistance thermometer mounted into a thermometer recess fabricated into the devices (recess labeled as RTD in Figure 2). This allowed the temperature to be measured as close as possible to the transducing resistances. 
For measurements the devices were preheated to within $\pm 1^{\circ} \mathrm{C}$ of the target and controlled to stay within this range throughout. Meanwhile, the PEG samples were pre-heated in a custom made dry-bath to the same temperature (Figure S2 (d), Supporting Information). The PEG solutions were then transferred to the device inlets using a pipette immediately prior to commencing the experiment. Each solution was tested three times, and three temperatures in total were tested (45 tests total). From the cone-plate rheometer measurement (Figure S1, Supporting Information) the absolute magnitude of the viscosity change was known over the temperature range examined. The absolute magnitude of the sample and control viscosities more than halved between 20 and $40{ }^{\circ} \mathrm{C}$. Despite this, the ratio of their viscosities remained constant — as such the expectation was that the bar-graph viscometer output should also remain constant. Indeed, as Figure 5(a) shows, the bar-graph type viscometer was able to sufficiently reduce temperature disturbances, and the results remained mostly consistent. In the elevated temperature tests, the precision, and accuracy of the device were somewhat reduced, likely due to higher temperature gradients, as well as generally lower viscosity. In general, temperature gradients in the device were difficult to avoid, as the upper device side had to be kept clear for recording of liquid movements. PEG and dye solutions will likely also diffuse at higher rates at the elevated temperatures of the test, resulting in a stronger blurring of the liquid front in the device. Conversely, when tested at reduced temperatures $\left(\sim 10^{\circ} \mathrm{C}\right)$, both the cFET and two-level trigger valve devices began to malfunction. The two-level trigger valves were often prematurely set off by condensation in the channel, and cFETs closed at extremely slow rates. The condensation was a particularly problematic because the testing stage and environmental temperature were different. If the general environmental temperature and chip temperature were identical, the dew point would be likely be lower. As such, condensation would be less of an issue in a real-world test.

Another potential disturbance force is the influence of hydrostatic pressure imbalance on the reported result. This was investigated and results are shown in Figure 5(b). In this test, unequal volumes of liquid were added to the sample and control inlets, respectively. Although the cFET prevents any fluid flow, hydrostatic pressure may still be transferred across the occluding bubble. As the radius of the inlets were known, this led to a determinable variation in liquid height in the inlets, and therefore in hydrostatic pressure applied to the sample. Although this configuration was not well controlled (due to the influence of inlet meniscus), it was considered sufficient to demonstrate whether an influence existed. As illustrated by the results shown in 
Figure 5(b), increasing hydrostatic pressure at the sample inlet decreased the flow in the corresponding resistance. While this trend is not yet fully understood, a potential explanation could be the relative pressure differential across the sample chamber. If the sample inlet reservoir is full to the same level as the water supply inlet, no hydrostatic pressure gradient exists across the sample reservoir. As a result, all flow in the sample chamber is directly caused by the capillary pump. On the other hand, when the sample inlet is empty, a hydrostatic pressure gradient develops across the reservoir. This pressure assists in the flow in the sample chamber, in addition to the influence of the capillary pump. Such an effect may create the unexpected negative relationship observed between hydrostatic pressure and fluid flow.

In addition to temperature and hydrostatic pressure, the effect of unequal clamping pressure on the device was also examined and demonstrated a moderate dependence of the result to unequal clamping force. This dependence was consistent with displacement of the PDMS roof into the transducing resistor creating a difference in resistance between the parallel transducers. Results of the clamping dependence are shown in Figure S5 (Supporting Information). It is expected that future versions of the viscometer fabricated and sealed using alternative manufacturing techniques, such as injection molding and thermal bonding, would not be prone to this type of disturbance.

In general, the two-liquid viscometer device described above functioned with good precision, and was able to reliably transduce the differential viscosity of samples over a wide range of relative (sample-to-control) and absolute viscosity values. For the future, the sample range will be extended towards biological fluids to target medical PoC applications.

\section{Read-out separation}

Regarding PoC applications, one shortcoming of the design presented so far is that the liquids must be colored, opaque, or dyed to be visually represented in the channel. Doing so may not be appropriate for all liquids one may wish to analyze in a PoC setting, with a notable example of this being blood plasma. ${ }^{[12]}$ This apparent limitation can however be easily circumvented by separating the transducing and read out functions of the viscometer. The latter is a particular strength of the capillaric circuit approach to viscometer devices, with a significant degree of flexibility enabled by the modular circuit-block-like design. In the following we present a device which utilizes four of the B1 type circuit blocks (Figure 2) to enable visualization of 
transparent liquids. This is achieved by adding a second B1 functional block, purely for visualization, in series to the transducing circuit block.

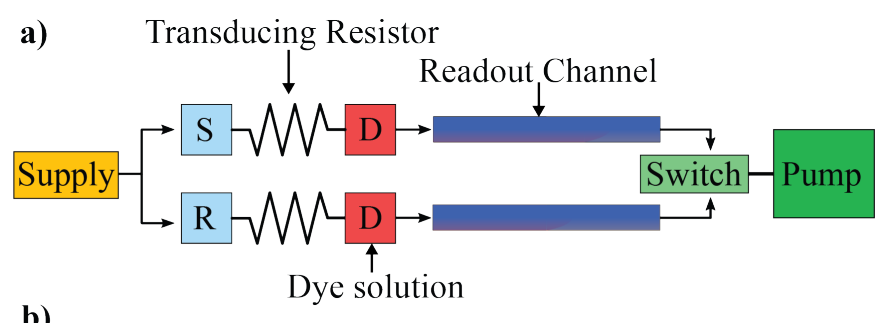

b)

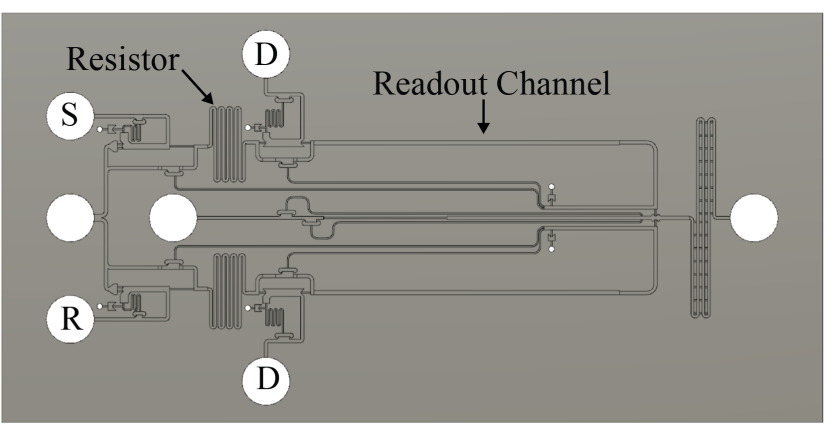

c)

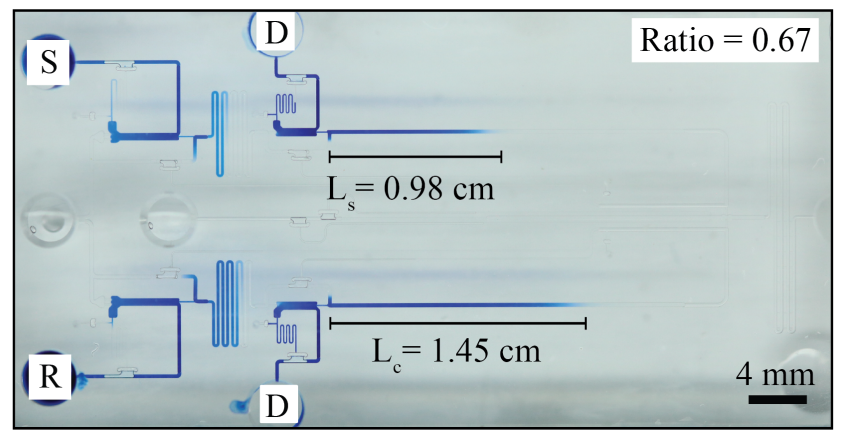

Figure 6: Read-out separated bar-graph type co-stream viscometer allowing transparent or translucent liquids to be measured. (a) Conceptual diagram showing the principle behind the device. An additional sample-intake flow resistance block is added between the transducing resistance and the connecting valve. (b) CAD rendering of the fabricated device. The shaded yellow areas are functionally identical to the previous device. (c) Photograph showing the function of the device with $4 \mathrm{kDa}$ and $2 \mathrm{kDa} P E G$ solutions as sample and control, respectively. Although intended for use with transparent liquids, the PEG samples were dyed for visualization purposes in this example.

A conceptual block diagram of this is shown in Figure 6(a), and as a fully realized capillaric circuit in Figure 6(b). This device operates identically to the previous device, except that now dye solution is loaded into the $\boldsymbol{D}$ inlet. Because the dye solution is in series with the transducing solution, the flow in the dye channels is still related to the rheological properties of the sample and control materials. As a result, the bar length readout can still be achieved with transparent liquids. 
The dual-reservoir differential viscometer device was tested with stock $2 \mathrm{kDa}$ PEG as control liquid, and stock $4 \mathrm{kDa}$ PEG as sample. Although the purpose of this design was to visualize the viscosity measurement of transparent samples, the PEG solutions were dyed in this test for visualization and verification purposes. The device functioned identically with transparent liquids in the sample inlets (not shown). A photograph of an example measurement is shown in Figure 6(c). As can be observed, the device successfully transduced the difference in viscosities as a change in bar lengths in the straight, dye-filled channels. It should be noted that in its current design, the device deviates even further from the true sample-to-control viscosity ratio. This is caused by the total increase in device resistance through the offset model, which was discussed above.

Just as with the previous device, it would be possible to manipulate the two-fluid or carrier fluid effect in a future device version by introducing a substantial source resistance between the transducing resistance and the sample chamber. This is especially important for biological fluids such as blood plasma, which have a viscosity range very close to that of water (in the range of $1.0-1.3 \mathrm{mPa} . \mathrm{s}$ at $\left.37^{\circ} \mathrm{C}\right) .{ }^{[12]}$ In this situation, the inaccuracy created by the working fluid offset would be near maximized by the closely matching rheological properties of the sample and working fluid. In this case, a highly resistive source resistance and transducing channel could be used with a very low resistance read-out channel. As before, the trade-off introduced by this pre-loading/source resistance approach relies on analyte self-pumping through large resistances. Ultimately, this would slow down the intake of viscous samples, and reduce the applicability of the circuit overall. As such, a compromise between experimental time, measurement accuracy and applicable analytes would need to be made depending on the specific application targeted.

For future applications, both the basic viscometer and separated bar-graph viscometer devices show great promise for reliably and quick transduction of rheological properties of bio-fluids. The basic device shows remarkable precision and there is a clear path to improve the accuracy through the flow resistance model. Finally, the flexibility and modularity of the basic circuit blocks illustrate that a wide range of fluids and measurands can be designed for with relative simplicity. As a next step, we will adjust the geometry of the device to render it suitable for biological analytes, such as blood and blood plasma, and investigate the measurement accuracy in this context. 


\section{Conclusion}

In this work a novel bar-graph co-stream viscometer device based capillary circuits was presented. The device successfully represented the rheological properties of Poly-(ethyleneglycol) samples and was internally consistent to within $2 \%$. In addition, it was able to achieve this performance without the use of external pumps, electronic transduction or the need for microscope-based optical measurements. Comparable to other co-stream viscometers, the bargraph type viscometer was able to compensate for temperature effects, as well as other disturbances such as manufacturing variations. This robust setup, as well as its self-driven design, make it ideally suited for use in PoC medical diagnostics. Due to its capillaric nature, the bar-graph type has advantages over other co-stream viscometers in its simplicity of readout, and the ability to add more functional blocks to the design. This functional block design will allow it to be applied to a wide range of potential areas beyond medical diagnostics.

In the future, we aim to further improve the accuracy and precision of the device through design calibration and advancement of the fabrication procedure and apply it to relevant biological analytes. 


\section{Experimental Methods}

\section{Device Fabrication:}

Capillaric devices were fabricated by CNC micro-milling channels into cast Poly-(MethylMethacrylate) PMMA sheets (4.5 mm general purpose acrylic; PSP Plastics, Christchurch, New Zealand) as previously reported. ${ }^{[9]}$ In brief, a Mini-Mill/GX micro milling machine with NSK3000 Spindle (Minitech Machinery Corporation, Norcross, GA, USA) was used for this purpose. The machining tools were purchased from Performance Micro Tool (Janesville, WI, USA) in diameters of $3.175 \mathrm{~mm}$ (SR-4-1250-S), $250 \mu \mathrm{m}$ (250M2X750S) and $100 \mu \mathrm{m}$ (100M2X300S) for the square heads and $200 \mu \mathrm{m}$ (TR-2-0080-BN) for the ball nose. The stock material was immersed in water during machining to improve the surface quality.

3D models, and CNC control code (G-code) were prepared using computer-aided design (CAD) software (Autodesk Fusion 360, 2021 Autodesk, Version 2.0.7421) for all functional units (CAD files provided in the Supporting Information. Note: $200 \mu \mathrm{m}$ channels are not depicted as round bottom in the files).

The machining parameters used were varied substantially depending on the structure being machined. Generally, chip-loads of approximately $8 \%$ were used for all cutters. The depth of cut was $50 \%$ tool diameter for all tools $<200 \mu \mathrm{m}$ diameter and $10 \%$ for tools $>200 \mu \mathrm{m}$ diameter.

After machining, the surface of the devices was coated with low molecular weight PMMA solution (average $\mathrm{Mw}=996,000,2.5 \%$ in Chlorobenzene, Sigma Aldrich, St Louis, MO, USA). Any remaining solvent was removed by drying samples at $90^{\circ} \mathrm{C}$ for $5 \mathrm{~min}$ on a hotplate. Finally, samples were plasma-treated ten times for $1 \mathrm{~min}$, each time at $15 \mathrm{~W}$, pulsed mode (ratio 50) using oxygen gas (3 sccm; Tergeo Plasma Cleaner, Pie Scientific, Union City, CA, USA) to remove all remaining solvent.

\section{Device testing:}

Prior to use, devices were rendered hydrophilic by exposure to oxygen plasma in a plasma asher (Pie Scientific, Tergeo, 2 x $1 \mathrm{~min}, 15 \mathrm{~W}, 15 / 255$ pulse ratio, $3 \mathrm{sscm} \mathrm{O}_{2}$ ). A Poly-(DiMethylSiloxane) - PDMS - (Sylgard 184, Electropar) layer acted as both a hydrophobic cover and a 
gasket for the microfluidic devices. The PDMS layer was mixed (10:1 w:w base:curing agent) and then cured at $80{ }^{\circ} \mathrm{C}$ for $2 \mathrm{~h}$ on a hotplate. The microfluidic device was sealed against the PDMS cover using a custom fabricated spring-loaded clamp, shown in Figure S2 (Supporting Information). The clamping force on the device was regulated by setting the displacement of four independent springs. This ensured that an even and repeatable pressure was applied to the device.

To measure the performance of the devices as a function of temperature, the testing platform (Figure S2, Supporting Information) integrated two Peltier modules into the base. The temperature differential across the Peltiers could be controlled by the input voltage. When the output heat-sink was immersed into an ice -bath, the temperature could be precisely controlled. A platinum resistance thermometer (RS-Pro, Class A, PT-100, 2 x 5.0mm, 611-7788) was mounted directly into a recess milled onto the device surface. This allowed for the temperature to be measured as close to the channel as possible. A variable bench-top power (Thurlby Thandar Instruments, EL302T, Triple Power Supply) was used to power the Peltier modules.

For testing, aqueous liquids were loaded into the inlets of the device using a pipette. An overhead camera (Canon EOS 760D, Canon Macro lens EF 100 mm 1:2.8 USM, 1080p, 25 fps) filmed the uptake of liquids into the device. Where visualization was required, various dyes were added to color the liquids (Tartrazine, Ponceau-4R, Brilliant Blue, Sigma Aldrich). ${ }^{[9]}$

The video data from the camera was converted uncompressed into raw avi format using ffmpeg (version 4.3.1, Copyright (c) 2000-2020 the FFmpeg developer) and analyzed using ImageJ (FIJI). ${ }^{[38]}$ The image processing comprised of background subtraction, contrast adjustment, binary color thresholding and average brightness extraction. Using the average brightness meant that the cropping of the image needed to be consistent between each experiment. The data extracted was then processed and plotted using Python 3.8.3 $3^{[42]}$. 


\section{Supporting Information}

Supporting Information is available from the Wiley Online Library or from the author.

\section{Acknowledgements}

The authors would like to thank Helen Devereux and Gary Turner of the Nanofabrication Laboratory at the University of Canterbury for technical support. Funding was provided by MBIE Grant UOCX1706. V. N. acknowledges Rutherford Discovery Fellowship RDF-19UOC-019 for additional funding. R.C.J.D acknowledges the New Zealand Royal Society Marsden Fund (contracts UOC1506 and UOC1910)

Received: ((will be filled in by the editorial staff))

Revised: ((will be filled in by the editorial staff)) Published online: ((will be filled in by the editorial staff))

\section{References}

[1] O. A. Soremekun, E. M. Datner, S. Banh, L. B. Becker, J. M. Pines, Am. J. Emerg. Med. 2013, 31, 291.

[2] C. Florkowski, A. Don-Wauchope, N. Gimenez, K. Rodriguez-Capote, J. Wils, A. Zemlin, Crit. Rev. Clin. Lab. Sci. 2017, 54, 471.

[3] P. A. Tideman, R. Tirimacco, D. P. Senior, J. J. Setchell, L. T. Huynh, R. Tavella, P. E. G. Aylward, D. P. B. Chew, Med. J. Aust. 2014, 200, 157.

[4] E. Lee-Lewandrowski, K. Lewandrowski, Clin. Lab. Med. 2009, 29, 479.

[5] K. Blattner, G. Nixon, S. Dovey, C. Jaye, J. Wigglesworth, Health Policy 2010, 96, 7.

[6] M. D. S. Shephard, B. C. Mazzachi, A. K. Shephard, T. Burgoyne, A. Dufek, J. A. Kit, D. Mills, D. Dunn, Point Care 2006, 5, 168.

[7] ISO/TC 212, "ISO 22870:2016," can be found under https://www.iso.org/cms/render/live/en/sites/isoorg/contents/data/standard/07/11/71119. html, 2016.

[8] R. Safavieh, D. Juncker, Lab. Chip 2013, 13, 4180.

[9] J. Menges, C. Meffan, F. Dolamore, C. Fee, R. Dobson, V. Nock, Lab. Chip 2021, 21, 205.

[10] G. D. O. Lowe, Ed. , Clinical Blood Rheology: Volume 1, CRC Press, Boca Raton, 2019.

[11] P. K. Ong, D. Lim, S. Kim, Crit. Rev. Biomed. Eng. 2010, 38, DOI 10.1615/CritRevBiomedEng.v38.i2.50.

[12] G. Késmárky, P. Kenyeres, M. Rábai, K. Tóth, Clin. Hemorheol. Microcirc. 2008, 39, 243.

[13] C. J. Pipe, G. H. McKinley, Mech. Res. Commun. 2009, 36, 110.

[14] N. Morhell, H. Pastoriza, Microfluid. Nanofluidics 2013, 15, 475.

[15] N. Srivastava, R. D. Davenport, M. A. Burns, Anal. Chem. 2005, 77, 383.

[16] N. Srivastava, M. A. Burns, Lab. Chip 2006, 6, 744.

[17] Y. Li, X. Men, G. Gao, Y. Tian, Y. Wen, X. Zhang, Lab. Chip 2021, 21, 719. 
[18] L. E. Rodd, J. J. Cooper-White, D. V. Boger, G. H. McKinley, J. Non-Newton. Fluid Mech. 2007, 143, 170.

[19] M. S. N. Oliveira, M. A. Alves, F. T. Pinho, G. H. McKinley, Exp. Fluids 2007, 43, 437.

[20] J. S. Lee, R. Dylla-Spears, N. P. Teclemariam, S. J. Muller, Appl. Phys. Lett. 2007, 90, 074103.

[21] P. E. Arratia, J. P. Gollub, D. J. Durian, Phys. Rev. E 2008, 77, 036309.

[22] S. E. Mena, Y. Li, J. McCormick, B. McCracken, C. Colmenero, K. Ward, M. A. Burns, Biomicrofluidics 2020, 14, 014109.

[23] J. Lee, A. Tripathi, Anal. Chem. 2005, 77, 7137.

[24] A. Bamshad, A. Nikfarjam, M. H. Sabour, Meas. Sci. Technol. 2018, 29, 095901.

[25] M. I. Mohammed, S. Haswell, I. Gibson, Procedia Technol. 2015, $20,54$.

[26] H. Hong, J. M. Song, E. Yeom, Biomicrofluidics 2019, 13, 014104.

[27] Y. J. Kang, S. Yang, Microfluid. Nanofluidics 2013, 14, 657.

[28] A. Olanrewaju, M. Beaugrand, M. Yafia, D. Juncker, Lab. Chip 2018, 18, 2323.

[29] S. Gupta, W. S. Wang, S. A. Vanapalli, Biomicrofluidics 2016, 10, 043402.

[30] T. Tian, J. Li, Y. Song, L. Zhou, Z. Zhu, C. James Yang, Lab. Chip 2016, 16, 1139.

[31] J. Xu, X. Hu, H. Khan, M. Tian, L. Yang, Anal. Chim. Acta 2019, 1071, 1.

[32] Y. Xie, X. Wei, Q. Yang, Z. Guan, D. Liu, X. Liu, L. Zhou, Z. Zhu, Z. Lin, C. Yang, Chem. Commun. 2016, 52, 13377.

[33] P. Shah, E. G. Freedman, Top. Cogn. Sci. 2011, 3, 560.

[34] R. C. Meffan, J. Menges, F. Dolamore, D. Mak, C. Fee, R. Dobson, V. Nock, 2021, DOI 10.33774/chemrxiv-2021-lnbml.

[35] C. Meffan, J. Menges, F. Dolamore, C. Fee, R. Dobson, V. Nock, in 2021 IEEE 34th Int. Conf. Micro Electro Mech. Syst. MEMS, 2021, pp. 218-221.

[36] B. J. Kirby, Micro- and Nanoscale Fluid Mechanics: Transport in Microfluidic Devices, Cambridge University Press, 2010.

[37] M. Zimmermann, H. Schmid, P. Hunziker, E. Delamarche, Lab. Chip 2007, 7, 119.

[38] C. A. Schneider, W. S. Rasband, K. W. Eliceiri, Nat. Methods 2012, 9, 671.

[39] K. V. Christ, B. B. Smith, F. E. Pfefferkorn, K. T. Turner, n.d., 8.

[40] A. Lashkaripour, R. Silva, D. Densmore, Microfluid. Nanofluidics 2018, 22, 31.

[41] D. Mikaelian, B. Jones, SN Appl. Sci. 2020, 2, 415.

[42] G. Van Rossum, F. Drake, Scotts Val. CA 2009. 
Claude Meffan, Julian Menges, Fabian Dolamore, Daniel Mak, Conan Fee, Volker M. Nock, Renwick C.J. Dobson

\section{A versatile capillaric microfluidics viscometer platform for bar-graph type point-of-care diagnostics}

A capillary action device has been design to measure the viscosity of fluids completely passively; without any electronics, external pumps or instrumentation. This design has applications for medical point-of-care testing and a range of other non-medical tests. In the future, we aim to use this device to measure the viscosity of blood and other medical analytes in point-of-care settings.

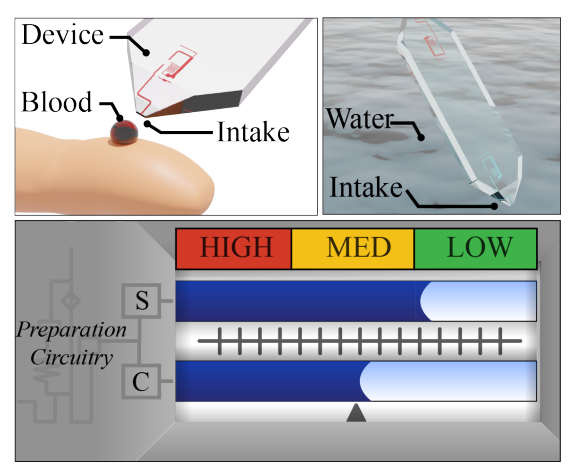

